\title{
LARGE SCALE STRUCTURE AND KINEMATICS OF THE ORION RIDGE
}

\author{
J. J. WISEMAN AND P. T. P. HO \\ Harvard-Smithsonian Center for Astrophysics
}

\section{SUMMARY}

Clumps and filamentary structures have previously been observed in the extended molecular ridge of OMC-1 (see below). The region is affected by multiple outflow components, shock emission that extends $0.5 \mathrm{pc}$ from the $\mathrm{BN} / \mathrm{KL}$ core, and a foreground HII region expanding into the cloud. The region also displays a complex kinematical character, with a large scale shift in radial velocity along the ridge, multiple cloud velocity components (Womack etal. 1993), and fast gradients across constituent cores (Harris et al. 1983, Wiseman and Ho 1993).

To clarify the structure and kinematics of the region, we have used the VLA to observe with high $\left(0.3 \mathrm{~km} \mathrm{~s}^{-1}\right)$ velocity resolution and high $\left(8^{\prime \prime}\right)$ angular resolution the $\mathrm{NH}_{3}(1,1)$ and $(2,2)$ rotation-inversion lines over 20 adjacent fields covering a $3^{\prime}$ by $8^{\prime}$ region encompassing the $\mathrm{KL}$ region. We present a linear mosaic of the 10 northern fields in Figure 1. The contours show the integrated intensity of the $(1,1)$ component. The greyscale shades portray the velocity field of the region by displaying the first moment integral of the same component. We find evidence of the extended clumpy filaments described by Murata et al. (1990) and Martín-Pintado etal. (1990). The fragments display velocity gradients in varying directions; some may be sites of young stars or collapsing cores which have not yet shed their angular momentum. We also find overlapping material with velocities differing by more than $2 \mathrm{~km} \mathrm{~s}^{-1}$ present within small projected areas. Such complicated kinematics are not well described by global rotation models for OMC-1.

The greyscale shades of Figure 2 portray the ratio of the $(2,2)$ to $(1,1)$ line intensity across part of the mosaicked region, with darker regions representing higher temperature. Contours are the integrated intensity of the $(1,1)$ line, as in Figure 1. Heating is apparent between core peaks, with temperatures approximately three times that of adjacent peaks in some regions. This effect could be the result of external heating from the foreground HII region which would show a greater impact on the regions of lower optical depth between core peaks. Even warmer regions are seen along filament edges, which could be the result of extended outflows shocking and heating the filament surfaces.

\section{REFERENCES}


Harris, A., Townes, C.II., Matsakis, D. N., and Palmer, P., 1983,Ap. J. (Lellers), , 265, L63

Martín-Pintado, J., Rodríguez-Franco, A., and Bachiller, R., 1990, Ap. J. (Letters), , 357, L49

Murata, Y., Kawabe, R., Ishiguro, M., Morita, K., Kasuga, T., Takano, T., Hawegawa, T., 1990, Ap. J., , 359, 125

Wiseman, J.J., and Ho, P. T. P., 1993, in preparation

Womack, M., Ziurys, L. M., and Sage, L. J., 1993, Ap. J., , to be published

Figure 1

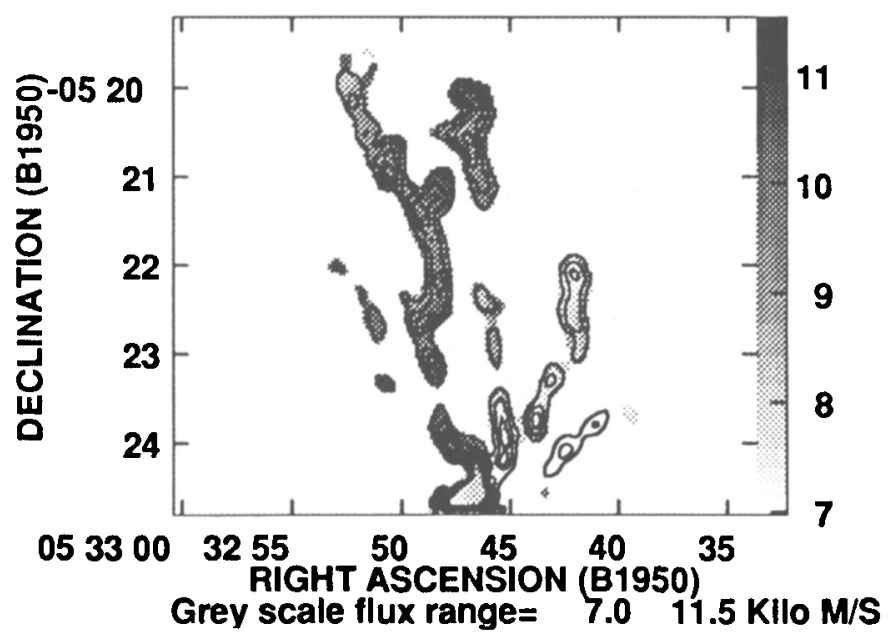

Figure 2

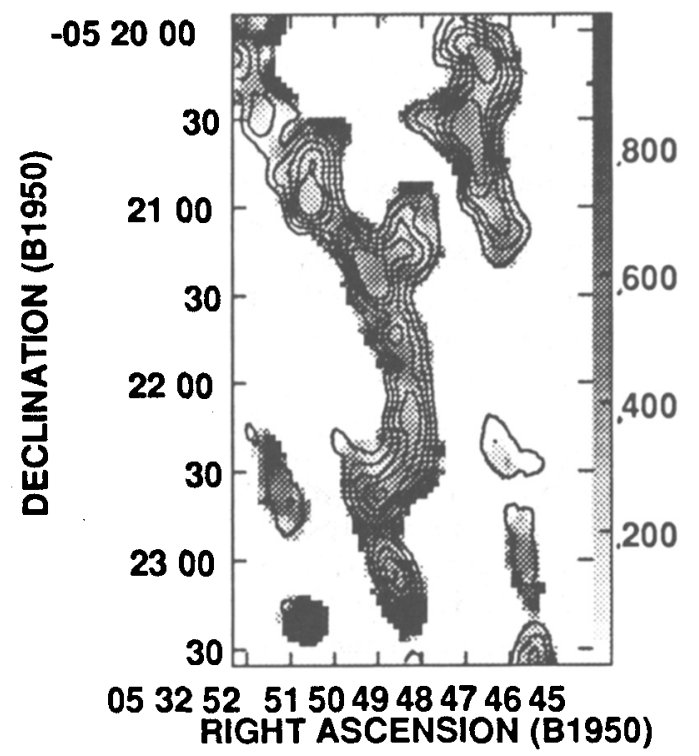

